\title{
Implementación de un prototipo de una dispensadora y envasadora de chocolates
}

\begin{abstract}
RESUMEN
El presente artículo describe la implementación del prototipo de una dispensadora y envasadora de chocolates, para ello se emplea técnicas de automatización para el control automático del proceso. El diseño del prototipo se realizó en un software CAD (Diseño asistido por computadora) y para implementación se requirió elementos neumáticos, sensores, un controlador lógico programable (PLC).Todos estos componentes se integraron a una faja transportadora.
\end{abstract}

Palabras clave: Implementación de un prototipo, automatización

IMPLEMENTATION OF A PROTOTYPE OF A DISPENSER OF CHOCOLATES

\section{ABSTRACT}

This article describes the implementation for a prototype of a chocolate filling and dispensing, using automation techniques for automatic control of the process. The design of the prototype was performed in a software CAD (computer aided design) and for the implementation was required pneumatic devices, sensors, programmable logic controller (PLC). All these components are integrated into a conveyor belt.

KEYWORDS: Implementation for a prototype, automation

\section{INTRODUCCIÓN}

El prototipo de la dispensadora y envasadora de chocolates es un sistema automatizado que consta de cuatro etapas: dispensador vertical de cajas, dispensador de chocolates, dispensador de tapas y taponadora de envases. El proceso es controlado por un PLC y funciona de manera automática con tan solo presionar un botón.

\section{DISEÑO DEL PROTOTIPO}

El diseño del prototipo de una dispensadora y envasadora de chocolates se realizó con la versión 2009 software Autocad para facilitar su desarrollo e implementación. Los principales elementos que componen el prototipo son:

\begin{tabular}{|l|c|c|}
\hline \multicolumn{1}{|c|}{ Descripción } & Cantidad & Especificación \\
\hline Planchas de aluminio & 2 & $1200 \times 100 \mathrm{~mm}$ \\
\hline Plancha acrílica & 1 & $600 \times 100 \mathrm{~mm}$ \\
\hline Perfiles de aluminio forma "I" & 2 & $25 \times 25 \times 3000 \mathrm{~mm}$ \\
\hline Perfiles de aluminio forma "T" & 2 & $25 \times 25 \times 3000 \mathrm{~mm}$ \\
\hline Perfiles de aluminio forma "L" & 2 & $25 \times 25 \times 3000 \mathrm{~mm}$ \\
\hline Perfiles de aluminio forma "U" & 2 & $50 \times 12 \times 3000 \mathrm{~mm}$ \\
\hline Tornillos & 100 & $1 / 8 \times 1 "$ \\
\hline $\begin{array}{l}\text { Pernos con tuerca y arandela } \\
\text { de presión }\end{array}$ & 50 & $5 / 32 \times 1$ " \\
\hline Remaches & 100 & $1 / 8 \times 1 / 4 "$ \\
\hline Frasco de plástico & 6 & $80 \times 80 \times 80 \mathrm{~mm}$ \\
\hline Tapa de plástico & 1 & $80 \times 80 \mathrm{~mm}$ \\
\hline Motor para faja transportadora & 1 & $220 \mathrm{VAC}$ \\
\hline $\begin{array}{l}\text { Motor de proceso de llenado } \\
\text { de envases }\end{array}$ & 3 & 12 VDC \\
\hline Electroválvulas neumáticas & 3 & 24 VDC \\
\hline Actuadores neumáticos & 3 & carrera 100mm \\
\hline Faja transportadora & 1 & $3 \mathrm{mt}$ \\
\hline $\begin{array}{l}\text { Tapón de jebe para sellado } \\
\text { de envase }\end{array}$ & 1 & ---- \\
\hline Eje de material inoxidable & 1 & $10 \times 100 \mathrm{~mm}$ \\
\hline Finales de carrera & 5 & 24 VDC \\
\hline Manguera neumática & $20 \mathrm{mt}$ & Diámetro 4mm \\
\hline Tornillo sin fin & 1 & $200 \mathrm{~mm}$ \\
\hline PLC & 1 & 8 inputs/8 outputs \\
\hline Cajitas de plástico & 5 & --- \\
\hline & & \\
\hline
\end{tabular}

* Magíster en Ingeniería Industrial. Jefe del Laboratorio de Automatización de la URP. E-mail: cim.urp@gmail. 


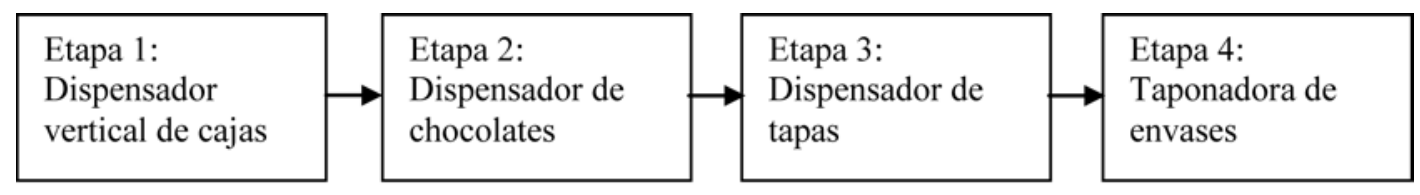

Figura 1: Etapas de la dispensadora y envasadora de chocolates.

\section{CARACTERÍSTICAS TÉCNICAS DEL PROTOTIPO}

Las características técnicas del prototipo son las siguientes:

\begin{tabular}{|l|l|}
\hline Capacidad del almacén & 10 envases \\
\hline Velocidad de la faja & $5 \mathrm{~cm} / \mathrm{seg}$ \\
\hline Tiempo de llenado de envases & $5 \mathrm{seg}$. \\
\hline Tipo de producto a envasarse & sólidos \\
\hline Dimensiones & $110 \times 35 \times 50 \mathrm{~cm}$ \\
\hline
\end{tabular}

\section{DESCRIPCIÓN DETALLADA DEL PROTOTIPO}

Las etapas que componen el proceso del prototipo de una dispensadora y envasadora de chocolates se aprecian en la Figura 1 y el prototipo en mención en la Figura 2.

\section{Dispensador vertical de cajas}

Es un almacén temporal de cajas. Posee cuatro perfiles de ángulo de aluminio, aquí se colocan manualmente cada una de las cajas que servirán de contenedores para los chocolates. En la parte inferior del dispensador está ubicado un cilindro neumático de doble efecto con una electroválvula $5 / 2$ monoestable. Cuando el proceso se inicia por medio de un pulsador eléctrico, la electroválvula se activa y permite que el cilindro se extienda, haciendo que la caja ubicada en la parte inferior del dispensador salga hacia la faja transportadora.

Cuando la caja es expulsada del dispensador se activa un final de carrera que está ubicado en la faja transportadora y permite que se active el motor que controla la faja. De esta manera, la caja se traslada por la faja transportadora hasta que active un segundo final de carrera que indica que el proceso se encuentra en su segunda etapa. La faja transportadora se detiene. Ver Figura 3.

\section{Dispensador de chocolates}

Está compuesta de una estructura de aluminio. Posee un motor de 24 VDC con un reductor de velocidad conectado mediante una polea a un tornillo sin fin. Sobre el tornillo van los chocolates. El tornillo al girar permite que caigan las golosinas una tras otra. A la salida del tornillo hay un canal y en su interior un final de carrera que detecta la cantidad de chocolates que van cayendo a la caja.

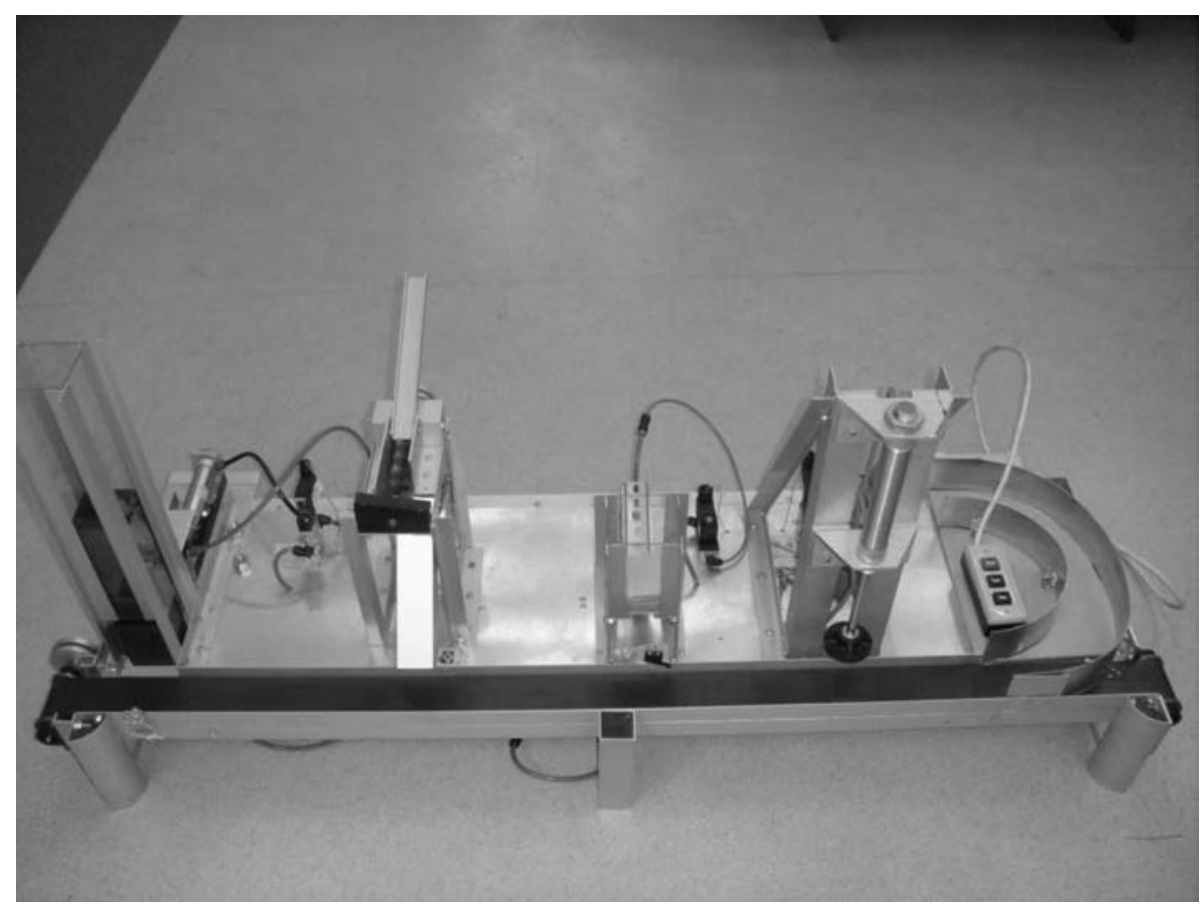

Figura 2: Prototipo de una dispensadora y envasadora de chocolates. 


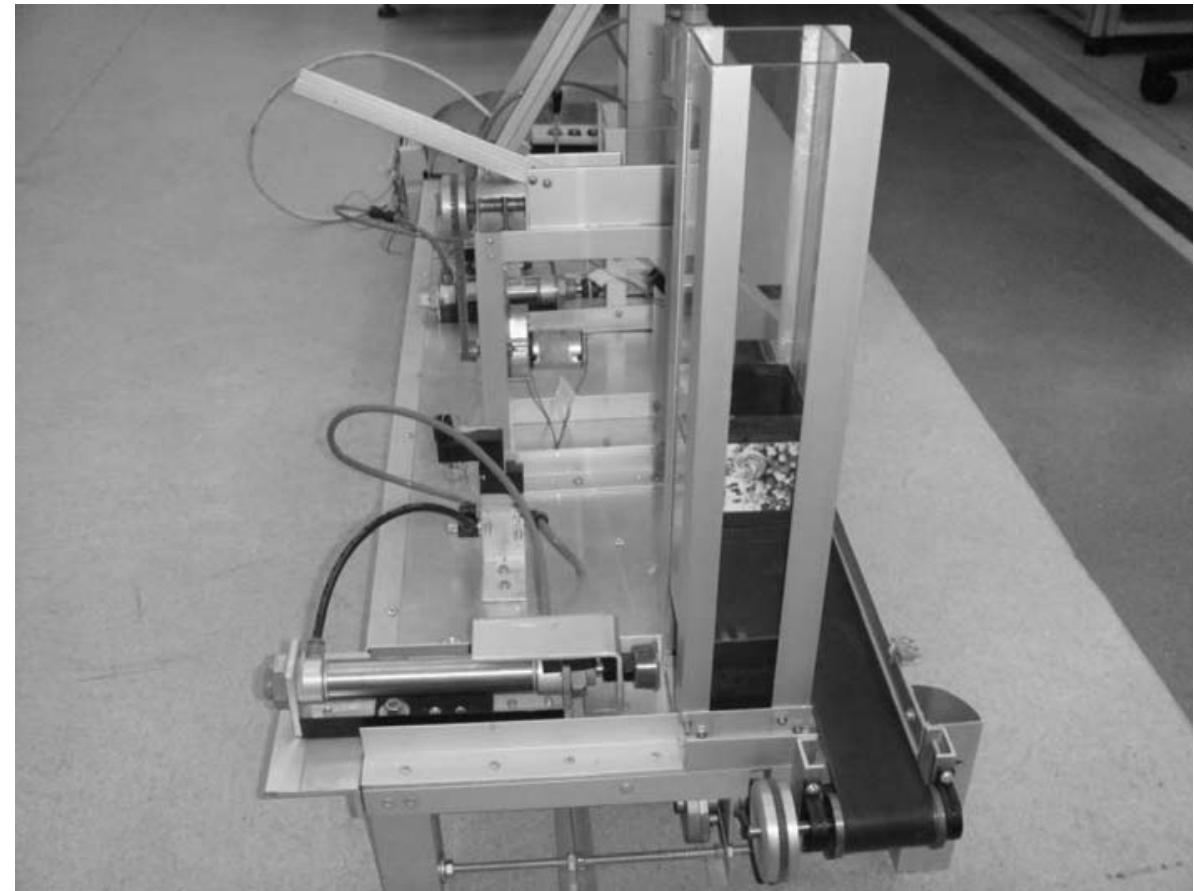

Figura 3: Dispensador vertical de cajas.

Cuando la cantidad de golosinas presentes en la caja sea la indicada (según programa), el tornillo deja de girar y el motor de la faja nuevamente se activa para transportar la caja hasta una siguiente etapa. Al costado de la faja hay un tercer final de carrera que al activarse hace que el motor se detenga, indicando que el proceso está en su tercera etapa. Ver Figura 4.

\section{Dispensador de tapas}

Está compuesta por una estructura de aluminio y en su pte inferior se ubica un cilindro neumático de simple efecto con una electroválvula $3 / 2$ monoestable. Cuando el tercer final de carrera se activa transcurre dos segundos y luego se activa la electroválvula permitiendo que el cilindro se extienda

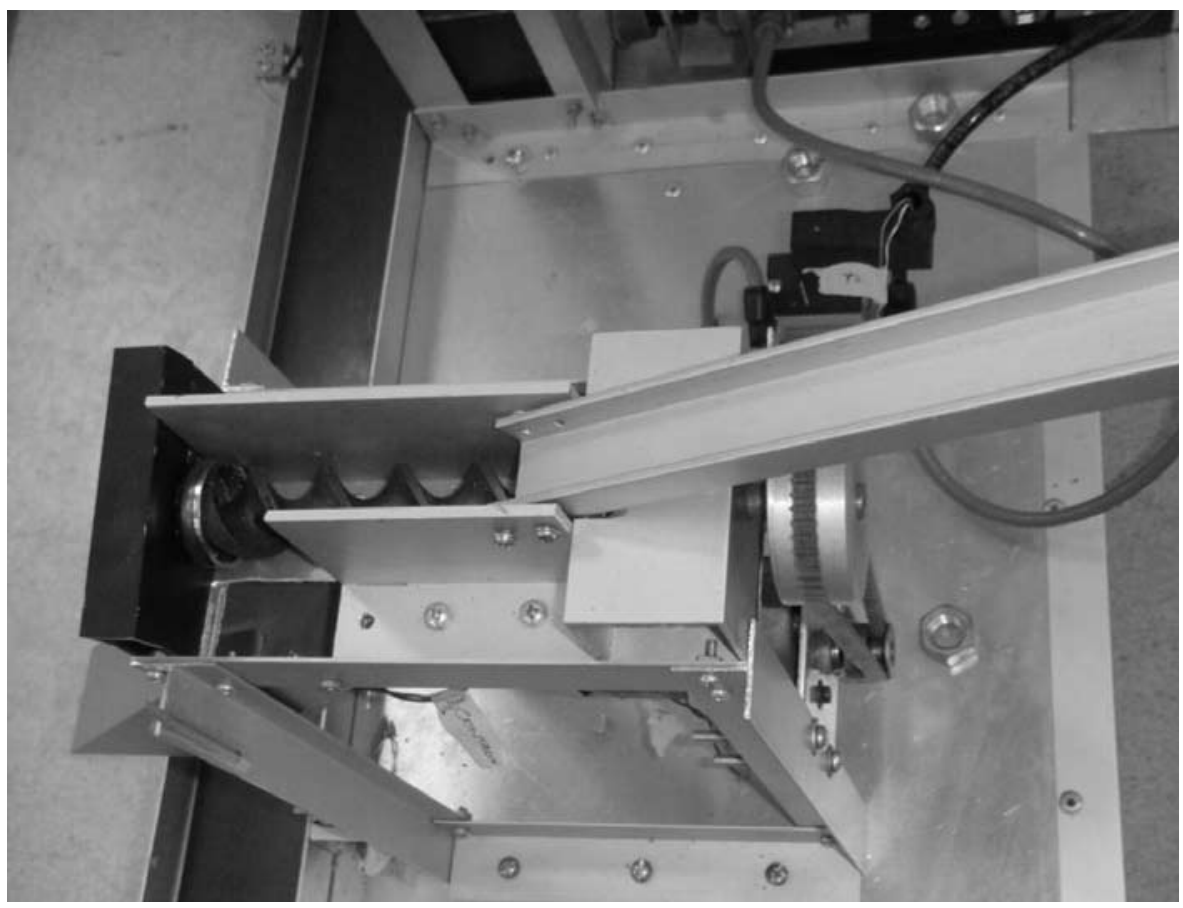

Figura 4: Dispensador de chocolates. 


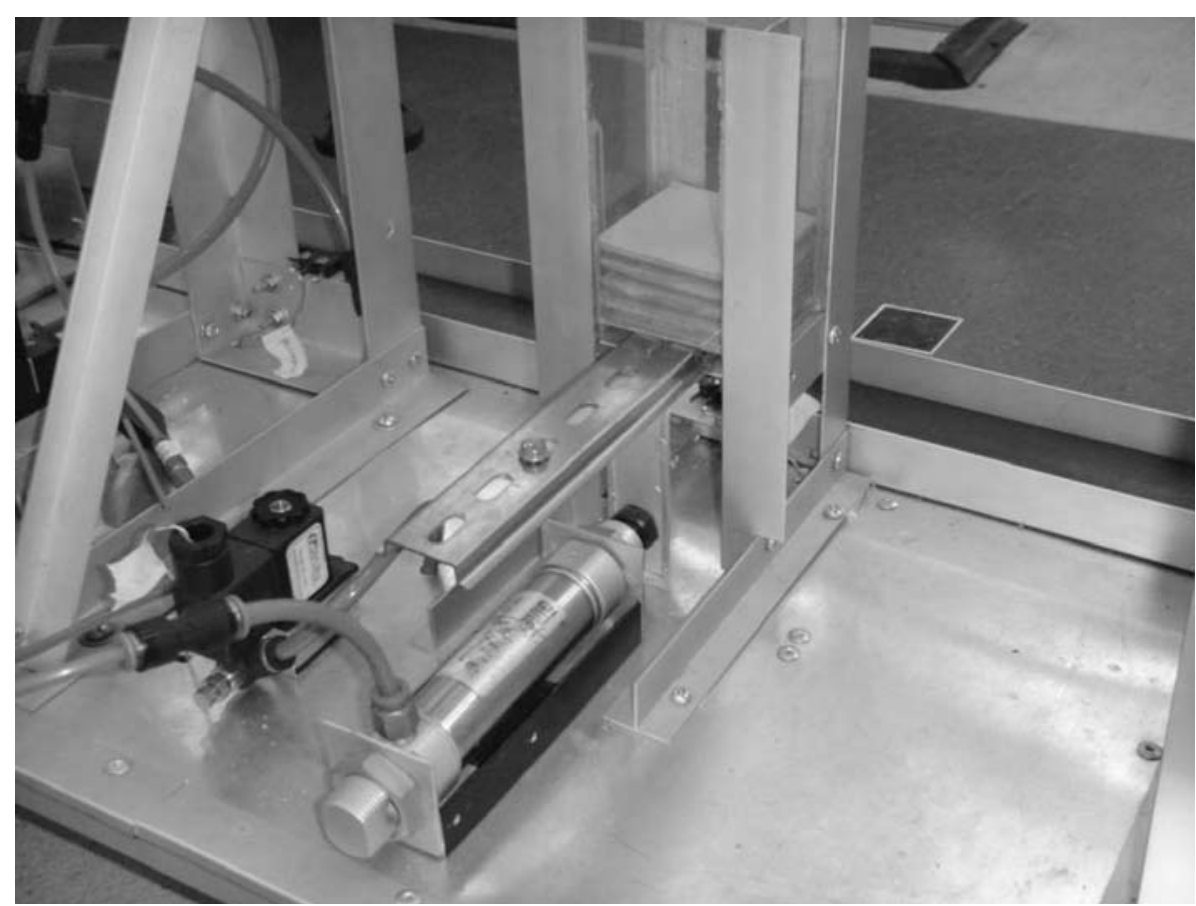

Figura 5: Dispensador de tapas.

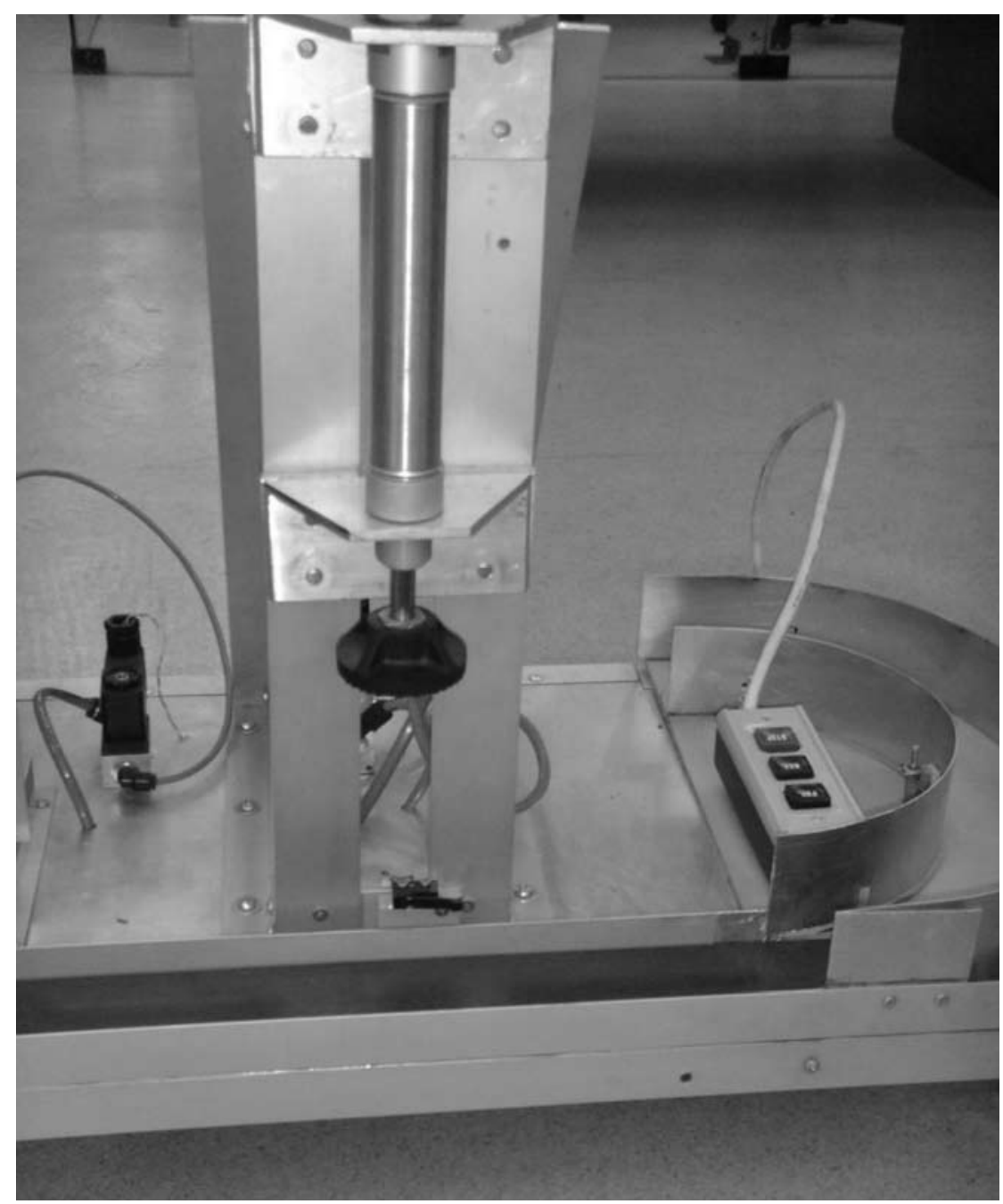

Figura 6. Taponadora de envase. 
para que una tapa caiga y se ubique sobre la caja que contiene los chocolates. En ese momento el cilindro se retrae y el motor nuevamente se activa permitiendo que la caja con tapa se traslade hacia la siguiente etapa. Un cuarto final de carrera indica que el proceso se encuentra en su cuarta etapa. Ver Figura 5.

\section{Taponadora de envase}

Está conformado por una estructura de aluminio sobre la cual se ubica un cilindro neumático de doble efecto en posición vertical, conectado a una electroválvula 5/2 monoestable. Al activarse el cuarto final de carrera este cilindro de doble efecto se extiende (baja el pistón) y logra realizar el taponado de la caja. Luego el cilindro se retrae (sube el pistón) y el motor de la faja nuevamente se activa para que la caja taponada que contiene los chocolates siga siendo transportada por la faja hacia la zona de productos terminados. Ver Figura 6.

\section{CONCLUSIONES}

1. El diseño del prototipo de una dispensadora y envasadora de chocolates permitió su implementación, con lo que se demostró que el proceso funciona correctamente.

2. Cada una de las etapas del proceso son controladas por un controlador lógico programable (PLC) al cual se conectan pulsadores, finales de carrera y electroválvulas.
3. EI PLC permite controlar los tiempos de activación de cada una de las etapas de proceso, así como también la cantidad de chocolates que irán dentro de la caja.

4. Aprovechando la información del proceso que obtiene y brinda el PLC, se podrá supervisar y controlar todo el proceso a través de una computadora mediante un sistema SCADA.

\section{REFERENCIAS BIBLIOGRÁFICAS}

Cembranos, F. J. (2002). Sistemas de control secuencial. Paraninfo, Madrid.

Deppert, W. y Stoll, K. (2001). Aplicaciones de la neumática. Alfaomega, México D.F.

Deppert, W. y Stoll, K. (2001). Dispositivos neumáticos. Alfaomega, México D.F.

Guillén Salvador, A. (1988). Aplicaciones industriales de la neumática. Marcombo, Barcelona.

Velásquez J., Noé C. (2000). Administración de recursos y control de procesos en el CIM. Perfiles de Ingeniería. Revista de la Facultad de Ingeniería de la Universidad Ricardo Palma, pp: 135-139.

Velásquez J. (2005). Los sensores en la producción. Revista de la Facultad de Ingeniería de la Universidad Ricardo Palma, pp: 112-116. 